\title{
On growth of meromorphic solutions for linear difference equations with meromorphic coefficients
}

Yanxia Liu*

"Correspondence:

cslyx@scut.edu.cn

School of Software Engineering,

South China University of

Technology, Panyu, Guangzhou,

510006, P.R. China

\begin{abstract}
In this paper, we consider the value distribution of meromorphic solutions for linear difference equations with meromorphic coefficients.
\end{abstract}

MSC: 30D35; 39A10

Keywords: difference equation; meromorphic coefficient; growth; zero

\section{Introduction and preliminaries}

Recently, several papers (including [1-7]) have been published regarding value distribution of meromorphic solutions of linear difference equations. We recall the following results. Chiang and Feng proved the following theorem.

Theorem A ([2]) Let $P_{0}(z), \ldots, P_{n}(z)$ be polynomials such that there exists an integer $l$, $0 \leq l \leq n$, such that

$$
\operatorname{deg}\left(P_{l}\right)>\max _{0 \leq j \leq n, j \neq l}\left\{\operatorname{deg}\left(P_{j}\right)\right\}
$$

holds. Suppose $f(z)$ is a meromorphic solution of the difference equation

$$
P_{n}(z) f(z+n)+\cdots+P_{1}(z) f(z+1)+P_{0}(z) f(z)=0 .
$$

Then we have $\sigma(f) \geq 1$.

In this paper, we use the basic notions of Nevanlinna's theory (see $[8,9])$. In addition, we use the notation $\sigma(f)$ to denote the order of growth of the meromorphic function $f(z)$, and $\lambda(f)$ to denote the exponent of convergence of zeros of $f(z)$.

Chen [1] weakened the condition (1.1) of Theorem A and proved the following results.

Theorem B ([1]) Let $P_{n}(z), \ldots, P_{0}(z)$ be polynomials such that $P_{n} P_{0} \not \equiv 0$ and

$$
\operatorname{deg}\left(P_{n}+\cdots+P_{0}\right)=\max \left\{\operatorname{deg} P_{j}: j=0, \ldots, n\right\} \geq 1 .
$$

Then every finite order meromorphic solution $f(z)(\not \equiv 0)$ of equation (1.2) satisfies $\sigma(f) \geq 1$, and $f(z)$ assumes every nonzero value $a \in \mathbb{C}$ infinitely often and $\lambda(f-a)=\sigma(f)$.

( 2013 Liu; licensee Springer. This is an Open Access article distributed under the terms of the Creative Commons Attribution License (http://creativecommons.org/licenses/by/2.0), which permits unrestricted use, distribution, and reproduction in any medium, provided the original work is properly cited. 
Theorem $\mathrm{C}([1])$ Let $F(z), P_{n}(z), \ldots, P_{0}(z)$ be polynomials such that $F P_{n} P_{0} \not \equiv 0$ and (1.3). Then every finite order transcendental meromorphic solution $f(z)$ of the equation

$$
P_{n}(z) f(z+n)+\cdots+P_{1}(z) f(z+1)+P_{0}(z) f(z)=F(z)
$$

satisfies $\sigma(f) \geq 1$ and $\lambda(f)=\sigma(f)$.

Theorem $\mathbf{D}([1])$ Let $F(z), P_{n}(z), \ldots, P_{0}(z)$ be polynomials such that $F P_{n} P_{0} \not \equiv 0$. Suppose that $f(z)$ is a meromorphic solution with infinitely many poles of (1.2) (or (1.4)). Then $\sigma(f) \geq 1$.

For the linear difference equation with transcendental coefficients

$$
A_{n}(z) f(z+n)+\cdots+A_{1}(z) f(z+1)+A_{0}(z) f(z)=0
$$

Chiang and Feng proved the following result.

Theorem $\mathbf{E}([2])$ Let $A_{0}(z), \ldots, A_{n}(z)$ be entire functions such that there exists an integer $l$, $0 \leq l \leq n$, such that

$$
\sigma\left(A_{l}\right)>\max \left\{\sigma\left(A_{j}\right): 0 \leq j \leq n, j \neq l\right\} .
$$

If $f(z)$ is a meromorphic solution of $(1.5)$, then we have $\sigma(f) \geq \sigma\left(A_{l}\right)+1$.

Laine and Yang proved the following theorem.

Theorem $\mathbf{F}$ ([5]) Let $A_{0}, \ldots, A_{n}$ be entire functions of finite order so that among those having the maximal order $\sigma:=\max \left\{\sigma\left(A_{k}\right): 0 \leq k \leq n\right\}$, exactly one has its type strictly greater than the others. Then for any meromorphic solution of

$$
A_{n}(z) f\left(z+C_{n}\right)+\cdots+A_{1}(z) f\left(z+C_{1}\right)+A_{0}(z) f(z)=0,
$$

we have $\sigma(f) \geq \sigma+1$.

Remark 1.1 If $A_{0}, \ldots, A_{n}$ are meromorphic functions satisfying (1.6), then Theorem $\mathrm{E}$ does not hold. For example, the equation

$$
y(z+1)-\left(e^{i}+\frac{e^{i}-1}{e^{i z}-1}\right) y(z)=0
$$

has a solution $y(z)=e^{i z}-1$, which $\sigma(y)=1<\sigma\left(A_{0}\right)+1$.

This example shows that for the linear difference equation with meromorphic coefficients, the condition (1.6) cannot guarantee that every transcendental meromorphic solution $f(z)$ of (1.7) satisfies $\sigma(f) \geq \sigma\left(A_{l}\right)+1$.

Thus, a natural question to ask is what conditions will guarantee every transcendental meromorphic solution $f(z)$ of (1.7) with meromorphic coefficients satisfies $\sigma(f) \geq$ $\sigma\left(A_{l}\right)+1$.

In this note, we consider this question and prove the following results. 
Theorem 1.1 Let $c_{1}, c_{2}\left(\neq c_{1}\right)$, a be nonzero constants, $h_{1}(z)$ be a nonzero meromorphic function with $\sigma\left(h_{1}\right)<1, B(z)$ be a nonzero meromorphic function.

If $B(z)$ satisfies any one of the following three conditions:

(i) $\sigma(B)>1$ and $\delta(\infty, B)>0$;

(ii) $\sigma(B)<1$;

(iii) $B(z)=h_{0}(z) e^{b z}$ where $b$ is a nonzero constant, $h_{0}(z)(\not \equiv 0)$ is a meromorphic function with $\sigma\left(h_{0}\right)<1$,

then every meromorphic solution $f(\not \equiv)$ of the difference equation

$$
f\left(z+c_{2}\right)+h_{1}(z) e^{a z} f\left(z+c_{1}\right)+B(z) f(z)=0
$$

satisfies $\sigma(f) \geq \max \{\sigma(B), 1\}+1$.

Further, if $\varphi(z)(\not \equiv 0)$ is a meromorphic function with

$$
\sigma(\varphi)<\max \{\sigma(B), 1\}+1
$$

then

$$
\lambda(f-\varphi)=\sigma(f) \geq \max \{\sigma(B), 1\}+1 .
$$

Corollary Under conditions of Theorem 1.1, every finite order solution $f(z)(\not \equiv 0)$ of $(1.8)$ has infinitely many fixed points, satisfies $\tau(f)=\sigma(f)$, and for any nonzero constant $c$,

$$
\lambda(f(z)-c)=\sigma(f) \geq \max \{\sigma(B), 1\}+1 .
$$

Example 1.1 The equation

$$
f(z+2)-\frac{1}{2} e^{2 z+3} f(z+1)-\frac{1}{2} e^{4 z+4} f(z)=0
$$

satisfies conditions of Theorem 1.1 and has a solution $f(z)=e^{z^{2}}$ satisfying $\lambda(f)=0$ and $\tau(f)=\sigma(f)=2$. This example shows that under conditions of Theorem 1.1, a meromorphic solution of (1.8) may have no zero.

Theorem 1.2 Let $h_{1}(z), c_{1}, c_{2}, a, B(z)$ satisfy conditions of Theorem 1.1, and let $F(z)(\not \equiv 0)$ be a meromorphic function with $\sigma(F)<\max \{\sigma(B), 1\}+1$. Then all meromorphic solutions with finite order of the equation

$$
f\left(z+c_{2}\right)+h_{1}(z) e^{a z} f\left(z+c_{1}\right)+B(z) f(z)=F(z)
$$

satisfy

$$
\lambda(f)=\sigma(f) \geq \max \{\sigma(B), 1\}+1
$$

with at most one possible exceptional solution with $\sigma(f)<\max \{\sigma(B), 1\}+1$.

Remark 1.2 Under conditions of Theorem 1.1, equation (1.8) has no rational solution. But equation (1.9) in Theorem 1.2 may have a rational solution. For example, the equation

$$
f(z+2)+e^{z} f(z+1)-e^{z} f(z)=z+2-e^{z}
$$


satisfies conditions of Theorem 1.2 and has a solution $f(z)=z$. This shows that in Theorem 1.2, there exists one possible exceptional solution with $\sigma(f)<\max \{\sigma(B), 1\}+1$.

\section{Proof of Theorem 1.1}

We need the following lemmas to prove Theorem 1.1.

Lemma $2.1([2,10])$ Given two distinct complex constants $\eta_{1}, \eta_{2}$, let $f$ be a meromorphic function of finite order $\sigma$. Then, for each $\varepsilon>0$, we have

$$
m\left(r, \frac{f\left(z+\eta_{1}\right)}{f\left(z+\eta_{2}\right)}\right)=O\left(r^{\sigma-1+\varepsilon}\right) .
$$

Lemma 2.2 (see [11]) Suppose that $P(z)=(\alpha+i \beta) z^{n}+\cdots(\alpha, \beta$ are real numbers, $|\alpha|+|\beta| \neq$ $0)$ is a polynomial with degree $n \geq 1$, that $A(z)(\not \equiv 0)$ is an entire function with $\sigma(A)<n$. Set $g(z)=A(z) e^{P(z)}, z=r e^{i \theta}, \delta(P, \theta)=\alpha \cos n \theta-\beta \sin n \theta$. Then, for any given $\varepsilon>0$, there exists a set $H_{1} \subset[0,2 \pi)$ that has the linear measure zero such that for any $\theta \in[0,2 \pi) \backslash\left(H_{1} \cup H_{2}\right)$, there is $R>0$ such that for $|z|=r>R$, we have that

(i) if $\delta(P, \theta)>0$, then

$$
\exp \left\{(1-\varepsilon) \delta(P, \theta) r^{n}\right\}<\left|g\left(r e^{i \theta}\right)\right|<\exp \left\{(1+\varepsilon) \delta(P, \theta) r^{n}\right\}
$$

(ii) if $\delta(P, \theta)<0$, then

$$
\exp \left\{(1+\varepsilon) \delta(P, \theta) r^{n}\right\}<\left|g\left(r e^{i \theta}\right)\right|<\exp \left\{(1-\varepsilon) \delta(P, \theta) r^{n}\right\},
$$

where $H_{2}=\{\theta \in[0,2 \pi) ; \delta(P, \theta)=0\}$ is a finite set.

Lemma 2.3 Let $c_{1}, c_{2}\left(\neq c_{1}\right)$, a be nonzero constants, $A_{j}(z)(j=0,1,2), F(z)$ be nonzero meromorphic functions. Suppose that $f(z)$ is a finite order meromorphic solution of the equation

$$
A_{2}(z) f\left(z+c_{2}\right)+A_{1}(z) f\left(z+c_{1}\right)+A_{0}(z) f(z)=F(z) .
$$

$$
\text { If } \sigma(f)>\max \left\{\sigma(F), \sigma\left(A_{j}\right)(j=0,1,2)\right\}, \text { then } \lambda(f)=\sigma(f) \text {. }
$$

Proof Suppose that $\sigma(f)=\sigma, \max \left\{\sigma(F), \sigma\left(A_{j}\right)(j=0,1,2)\right\}=\alpha$. Then $\sigma>\alpha$. Equation (2.3) can be rewritten as the form

$$
\frac{1}{f(z)}=\frac{F(z)}{f(z)}\left(A_{2}(z) \frac{f\left(z+c_{2}\right)}{f(z)}+A_{1}(z) \frac{f\left(z+c_{1}\right)}{f(z)}+A_{0}(z)\right) .
$$

Thus, by (2.4), we deduce that

$$
\begin{aligned}
T(r, f) & =T\left(r, \frac{1}{f}\right)+O(1) \\
& =m\left(r, \frac{1}{f}\right)+N\left(r, \frac{1}{f}\right)+O(1)
\end{aligned}
$$




$$
\begin{aligned}
\leq & N\left(r, \frac{1}{f}\right)+m\left(r, \frac{1}{F}\right)+\sum_{j=0}^{2} m\left(r, A_{j}\right) \\
& +m\left(r, \frac{f\left(z+c_{2}\right)}{f(z)}\right)+m\left(r, \frac{f\left(z+c_{1}\right)}{f(z)}\right)+O(1) .
\end{aligned}
$$

For any given $\varepsilon\left(0<\varepsilon<\min \left\{\frac{1}{4}, \frac{\sigma-\alpha}{4}\right\}\right)$, and for sufficiently large $r$, we have that

$$
m\left(r, \frac{1}{F}\right) \leq T(r, F) \leq r^{\alpha+\varepsilon}, \quad m\left(r, A_{j}\right) \leq r^{\alpha+\varepsilon} \quad(j=0,1,2)
$$

By Lemma 2.1, we obtain

$$
m\left(r, \frac{f\left(z+c_{2}\right)}{f(z)}\right) \leq M r^{\sigma-1+\varepsilon} \text { and } m\left(r, \frac{f\left(z+c_{1}\right)}{f(z)}\right) \leq M r^{\sigma-1+\varepsilon}
$$

where $M(>0)$ is some constant.

By $\sigma(f)=\sigma$, there exists a sequence $\left\{r_{n}\right\}$ satisfying $r_{1}<r_{2}<\cdots, r_{n} \rightarrow \infty$ such that

$$
\lim _{n \rightarrow \infty} \frac{\log T\left(r_{n}, f\right)}{\log r_{n}}=\sigma
$$

Thus, for sufficiently large $r_{n}$, we have that

$$
T\left(r_{n}, f\right) \geq r_{n}^{\sigma-\varepsilon}
$$

Substituting (2.6)-(2.9) into (2.5), we obtain for sufficiently large $r_{n}$

$$
r_{n}^{\sigma-\varepsilon} \leq T\left(r_{n}, f\right) \leq N\left(r_{n}, \frac{1}{f}\right)+4 r_{n}^{\alpha+\varepsilon}+2 M r_{n}^{\sigma-1+\varepsilon}
$$

Since $\varepsilon<\min \left\{\frac{1}{4}, \frac{\sigma-\alpha}{4}\right\}$ and $\varepsilon$ is arbitrary, by (2.10), we obtain

$$
\varlimsup_{n \rightarrow \infty} \frac{\log N\left(r_{n}, \frac{1}{f}\right)}{\log r_{n}}=\sigma
$$

Hence, $\lambda(f)=\sigma(f)=\sigma$.

Proof of Theorem 1.1 Suppose that $f(z)(\not \equiv 0)$ is a meromorphic solution of equation (1.8) with $\sigma(f)<\infty$.

(1) Suppose that $B(z)$ satisfies the condition (i): $\sigma(B)>1$ and $\delta(\infty, B)=\delta>0$. Thus, for sufficiently large $r$,

$$
m(r, B)>\frac{\delta}{2} T(r, B)
$$

Clearly, $\sigma(f) \geq \sigma(B)$ by (1.8). By Lemma 2.1, we see that for any given $\varepsilon\left(0<\varepsilon<\frac{\sigma(B)-1}{3}\right)$,

$$
m\left(r, \frac{f\left(z+c_{j}\right)}{f(z)}\right)=O\left(r^{\sigma(f)-1+\varepsilon}\right) \quad(j=1,2)
$$


and

$$
m\left(r, h_{1}(z) e^{a z}\right) \leq T\left(r, h_{1}(z) e^{a z}\right) \leq r^{1+\varepsilon}
$$

By (1.8), we have that

$$
-B(z)=\frac{f\left(z+c_{2}\right)}{f(z)}+h_{1}(z) e^{a z} \frac{f\left(z+c_{1}\right)}{f(z)} .
$$

Substituting (2.11)-(2.13) into (2.14), we deduce that

$$
\begin{aligned}
\frac{\delta}{2} T(r, B) & \leq m(r, B) \\
& \leq m\left(r, h_{1}(z) e^{a z}\right)+m\left(r, \frac{f\left(z+c_{2}\right)}{f(z)}\right)+m\left(r, \frac{f\left(z+c_{1}\right)}{f(z)}\right) \\
& \leq r^{1+\varepsilon}+O\left(r^{\sigma(f)-1+\varepsilon}\right) .
\end{aligned}
$$

By $\sigma(B)=\sigma$, there is a sequence $r_{j}\left(1<r_{1}<r_{2}<\cdots, r_{j} \rightarrow \infty\right)$ satisfying

$$
T\left(r_{j}, B\right)>r_{j}^{\sigma(B)-\varepsilon}
$$

Thus, by (2.15) and (2.16), we obtain

$$
\frac{\delta}{2} r_{j}^{\sigma(B)-\varepsilon} \leq r_{j}^{1+\varepsilon}+M r_{j}^{\sigma(f)-1+\varepsilon}
$$

where $M(>0)$ is some constant. Combining (2.17) and $\varepsilon<\frac{\sigma(B)-1}{3}$, it follows that

$$
\frac{\delta}{2} r_{j}^{\sigma(B)-\varepsilon}(1+o(1)) \leq M r_{j}^{\sigma(f)-1+\varepsilon}
$$

So that, it follows that $\sigma(f) \geq \sigma(B)+1=\max \{\sigma(B), 1\}+1$.

(2) Suppose that $B(z)$ satisfies the condition (ii): $\sigma(B)<1$. Using the same method as in (1), we can obtain $\sigma(f) \geq \max \{\sigma(B), 1\}+1$.

(3) Suppose that $B(z)$ satisfies the condition (iii): $B(z)=h_{0}(z) e^{b z}$, where $b$ is a nonzero constant, $h_{0}(z)(\not \equiv 0)$ is a meromorphic function with $\sigma\left(h_{0}\right)<1$.

Now we need to prove $\sigma(f) \geq 2$. Contrary to the assertion, suppose that $\sigma(f)=\alpha<2$. We will deduce a contradiction. Set $z=r e^{i \theta}$. Then

$$
\left\{\begin{array}{l}
\boldsymbol{\operatorname { R e }}\{a z\}=\delta(a z, \theta)|a| r=|a| r \cos (\arg a+\theta), \\
\boldsymbol{\operatorname { R e }}\{b z\}=\delta(b z, \theta)|b| r=|b| r \cos (\arg b+\theta) .
\end{array}\right.
$$

In what follows, we divide this proof into three subcases: (a) $\arg a \neq \arg b$; (b) $\arg a=\arg b$ and $|a| \neq|b|$; (c) $a=b$.

Subcase (a). Since $\arg a \neq \arg b$ and (2.18), it is easy to see that there exists a ray $\arg z=\theta_{0}$ such that

$$
\left\{\begin{array}{l}
\boldsymbol{\operatorname { R e }}\{a z\}=\delta\left(a z, \theta_{0}\right)|a| r=|a| r \cos \left(\arg a+\theta_{0}\right)<0, \\
\boldsymbol{\operatorname { R e }}\{b z\}=\delta\left(b z, \theta_{0}\right)|b| r=|b| r \cos \left(\arg b+\theta_{0}\right)>0 .
\end{array}\right.
$$


By (1.8) and (2.19), we see that $f(z)$ cannot be a rational function. By Lemma 2.1, (2.12) holds. By Lemma 2.2 and (2.19), it is easy to see that for any given $\varepsilon_{1}\left(0<\varepsilon_{1}<\min \left\{\frac{1}{2}, \frac{2-\alpha}{2}\right\}\right)$ and for sufficiently large $r$,

$$
\left|h_{0}\left(r e^{i \theta_{0}}\right) e^{b r e^{i \theta_{0}}}\right| \geq \exp \left\{\left(1-\varepsilon_{1}\right)|b| \delta\left(b z, \theta_{0}\right) r\right\},
$$

and

$$
\left|h_{1}\left(r e^{i \theta_{0}}\right) e^{a r e^{i \theta_{0}}}\right| \leq \exp \left\{\left(1-\varepsilon_{1}\right)|a| \delta\left(a z, \theta_{0}\right) r\right\}<1 .
$$

Thus, by (1.8), (2.12), (2.20) and (2.21), we deduce that

$$
\begin{aligned}
\exp \left\{\left(1-\varepsilon_{1}\right)|b| \delta\left(b z, \theta_{0}\right) r\right\} & \leq\left|h_{0}\left(r e^{i \theta_{0}}\right) e^{b r e^{i \theta_{0}}}\right| \\
& \leq\left|\frac{f\left(r e^{i \theta_{0}}+c_{2}\right)}{f\left(r e^{i \theta_{0}}\right)}\right|+\left|h_{1}\left(r e^{i \theta_{0}}\right) e^{a r e^{i \theta_{0}}}\right|\left|\frac{f\left(r e^{i \theta_{0}}+c_{1}\right)}{f\left(r e^{i \theta_{0}}\right)}\right| \\
& \leq 2 \exp \left\{r^{\sigma(f)-1+\varepsilon_{1}}\right\} .
\end{aligned}
$$

By $\delta\left(b z, \theta_{0}\right)=\cos \left(\arg b+\theta_{0}\right)>0, \sigma(f)=\alpha<2$ and $\varepsilon_{1}<\frac{2-\alpha}{2}$, it is easy to see that (2.22) is a contradiction. Hence, $\sigma(f) \geq 2$.

Subcase (b). By $\arg a=\arg b$ and $|a| \neq|b|$, we see that $f(z)$ cannot be a rational function. By Lemma 2.1, (2.12) holds. By $\arg a=\arg b$ and (2.18), we take $\theta_{1}=-\arg a$, then $\delta\left(a z, \theta_{1}\right)=$ $\delta\left(b z, \theta_{1}\right)=1$ and

$$
\boldsymbol{\operatorname { R e }}\left\{a r e^{i \theta_{1}}\right\}=|a| r \quad \text { and } \quad \boldsymbol{\operatorname { R e }}\left\{b r e^{i \theta_{1}}\right\}=|b| r .
$$

Now suppose that $|b|>|a|$. By Lemma 2.2, for any given $\varepsilon_{2}\left(0<\varepsilon_{2}<\min \left\{2-\alpha, \frac{|b|-|a|}{2(|b|+|a|)}\right\}\right)$,

$$
\left|h_{0}\left(r e^{i \theta_{1}}\right) e^{b r e^{i \theta_{1}}}\right| \geq \exp \left\{\left(1-\varepsilon_{2}\right)|b| r\right\}
$$

and

$$
\left|h_{1}\left(r e^{i \theta_{1}}\right) e^{a r e^{i \theta_{1}}}\right| \leq \exp \left\{\left(1+\varepsilon_{2}\right)|a| r\right\} .
$$

Thus, by (1.8), (2.12), (2.24) and (2.25), we deduce that

$$
\begin{aligned}
\exp \left\{\left(1-\varepsilon_{2}\right)|b| r\right\} & \leq\left|h_{0}\left(r e^{i \theta_{1}}\right) e^{b r e^{i \theta_{1}}}\right| \\
& \leq\left|\frac{f\left(r e^{i \theta_{1}}+c_{2}\right)}{f\left(r e^{i \theta_{1}}\right)}\right|+\left|h_{1}\left(r e^{i \theta_{1}}\right) e^{a r e^{i \theta_{1}}}\right|\left|\frac{f\left(r e^{i \theta_{1}}+c_{1}\right)}{f\left(r e^{i \theta_{1}}\right)}\right| \\
& \leq \exp \left\{r^{\sigma(f)-1+\varepsilon_{2}}\right\}+\exp \left\{\left(1+\varepsilon_{2}\right)|a| r\right\} \exp \left\{r^{\sigma(f)-1+\varepsilon_{2}}\right\} .
\end{aligned}
$$

Since $\varepsilon_{2}<2-\alpha$, we have that $\sigma(f)-1+\varepsilon_{2}=\alpha-1+\varepsilon_{2}<1$. Combining this and (2.26), we obtain

$$
\exp \left\{\left(1-\varepsilon_{2}\right)|b| r\right\}<\exp \left\{\left(1+\varepsilon_{2}\right)|a| r(1+o(1))\right\}(1+o(1)) \text {. }
$$

By $\varepsilon_{2}<\frac{|b|-|a|}{2(|b|+|a|)}$, we see that (2.27) is a contradiction. 
Now suppose that $|b|<|a|$. Using the same method as above, we can also deduce a contradiction.

Hence, $\sigma(f) \geq 2$ in Subcase (b).

Subcase (c). We first affirm that $f(z)$ cannot be a nonzero rational function. In fact, if $f(z)$ is a rational function, then $e^{a z}\left[h_{1}(z) f\left(z+c_{1}\right)+h_{0}(z) f(z)\right]=-f\left(z+c_{2}\right)$ is a rational function. So that $h_{1}(z) f\left(z+c_{1}\right)+h_{0}(z) f(z) \equiv 0$, that is, $f\left(z+c_{2}\right) \equiv 0$, a contradiction.

By Lemma 2.1, (2.12) holds. By $a=b$, equation (1.8) can be rewritten as

$$
e^{-a z} f\left(z+c_{2}\right)+h_{1}(z) f\left(z+c_{1}\right)+h_{0}(z) f(z)=0 .
$$

Using the same method as in the proof of (1), we can obtain $\sigma(f) \geq 2$.

(4) Suppose that $\varphi(z)(\not \equiv 0)$ is a meromorphic function with $\sigma(\varphi)<\max \{\sigma(B), 1\}+1$. Set $g(z)=f(z)-\varphi(z)$. Substituting $f(z)=g(z)+\varphi(z)$ into (1.8), we obtain

$$
\begin{aligned}
& g\left(z+c_{2}\right)+h_{1}(z) e^{a z} g\left(z+c_{1}\right)+B(z) g(z) \\
& \quad=-\left[\varphi\left(z+c_{2}\right)+h_{1}(z) e^{a z} \varphi\left(z+c_{1}\right)+B(z) \varphi(z)\right] .
\end{aligned}
$$

If $\varphi\left(z+c_{2}\right)+h_{1}(z) e^{a z} \varphi\left(z+c_{1}\right)+B(z) \varphi(z) \equiv 0$, then $\varphi(z)$ is a nonzero meromorphic solution of (1.8). Thus, by the proof above, we have that $\sigma(\varphi) \geq \max \{\sigma(B), 1\}+1$. This contradicts our condition that $\sigma(\varphi)<\max \{\sigma(B), 1\}+1$. Hence, $\varphi(z+2)+h_{1}(z) e^{a z} \varphi(z+1)+B(z) \varphi(z) \not \equiv 0$, and

$$
\sigma\left(\varphi\left(z+c_{2}\right)+h_{1}(z) e^{a z} \varphi\left(z+c_{1}\right)+B(z) \varphi(z)\right)<\max \{\sigma(B), 1\}+1 \leq \sigma(f)=\sigma(g) .
$$

Applying this and Lemma 2.3 to (2.29), we deduce that

$$
\lambda(f-\varphi)=\lambda(g)=\sigma(g) \geq \max \{\sigma(B), 1\}+1 .
$$

Thus, Theorem 1.1 is proved.

\section{Proof of Theorem 1.2}

Suppose that $f_{0}$ is a meromorphic solution of (1.9) with

$$
\sigma\left(f_{0}\right)<\max \{\sigma(B), 1\}+1
$$

If $f^{*}(z)\left(\not \equiv f_{0}(z)\right)$ is another meromorphic solution of $(1.9)$ satisfying $\sigma\left(f^{*}\right)<\max \{\sigma(B), 1\}+$ 1 , then

$$
\sigma\left(f^{*}-f_{0}\right)<\max \{\sigma(B), 1\}+1
$$

But $f^{*}-f_{0}$ is a solution of the corresponding homogeneous equation (1.8) of (1.9). By Theorem 1.1, we have $\sigma\left(f^{*}-f_{0}\right) \geq \max \{\sigma(B), 1\}+1$, a contradiction. Hence equation (1.9) possesses at most one exceptional solution $f_{0}$ with $\sigma\left(f_{0}\right)<\max \{\sigma(B), 1\}+1$.

Now suppose that $f$ is a meromorphic solution of (1.9) with

$$
\max \{\sigma(B), 1\}+1 \leq \sigma(f)<\infty
$$


Since $\sigma(f)>\max \left\{\sigma(B), \sigma(F), \sigma\left(h(z) e^{a z}\right)\right\}$, applying Lemma 2.3 to (1.9), we obtain

$$
\lambda(f)=\sigma(f) .
$$

Thus, Theorem 1.2 is proved.

\section{Competing interests}

The author declares that they have no competing interests.

\section{Acknowledgements}

The author is grateful to the referees for a number of helpful suggestions to improve the paper. This research was partly supported by the National Natural Science Foundation of China (grant no. 11171119).

Received: 2 December 2012 Accepted: 26 February 2013 Published: 19 March 2013

\section{References}

1. Chen, ZX: Growth and zeros of meromorphic solution of some linear difference equations. J. Math. Anal. Appl. 373, 235-241 (2011)

2. Chiang, YM, Feng, SJ: On the Nevanlinna characteristic of $f(z+\eta)$ and difference equations in the complex plane. Ramanujan J. 16, 105-129 (2008)

3. Ishizaki, K: On difference Riccati equations and second order linear difference equations. Aequ. Math. 81, 185-198 (2011)

4. Ishizaki, K, Yanagihara, N: Wiman-Valiron method for difference equations. Nagoya Math. J. 175, 75-102 (2004)

5. Laine, I: Nevanlinna Theory and Complex Differential Equations. de Gruyter, Berlin (1993)

6. Li, S, Gao, ZS: Finite order meromorphic solutions of linear difference equations. Proc. Jpn Acad., Ser. A 87(5), 73-76 (2011)

7. Zheng, XM, Tu, J: Growth of meromorphic solutions of linear difference equations. J. Math. Anal. Appl. 384, 349-356 (2011)

8. Hayman, WK: Meromorphic Functions. Clarendon, Oxford (1964)

9. Laine, I, Yang, CC: Clunie theorems for difference and q-difference polynomials. J. Lond. Math. Soc. 76(3), 556-566 (2007)

10. Halburd, RG, Korhonen, R: Difference analogue of the lemma on the logarithmic derivative with applications to difference equations. J. Math. Anal. Appl. 314, 477-487 (2006)

11. Chen, ZX: The growth of solutions of $f^{\prime \prime}+e^{-z} f^{\prime}+Q(z) f=0$ where the order $(Q)=1$. Sci. China Math. 45(3), 290-300 (2002)

doi:10.1186/1687-1847-2013-60

Cite this article as: Liu: On growth of meromorphic solutions for linear difference equations with meromorphic coefficients. Advances in Difference Equations 2013 2013:60.

\section{Submit your manuscript to a SpringerOpen ${ }^{\circ}$ journal and benefit from:}

- Convenient online submission

Rigorous peer review

- Immediate publication on acceptance

- Open access: articles freely available online

- High visibility within the field

- Retaining the copyright to your article 